Systematic Review

\title{
Comparative Effectiveness of Suprascapular Nerve Block in the Relief of Acute Post-Operative Shoulder Pain: A Systematic Review and Meta-analysis
}

\author{
Ke-Vin Chang, MD, PhD', Wei-ting Wu, MD², Chen-Yu Hung, MD', \\ Der-Sheng Han, MD, PhD' ${ }^{1}$, Rong-Sen Yang, MD³, PhD, Chung-Hsun Chang, MD ${ }^{4}$, \\ and Chih-Peng Lin, MD ${ }^{4}$
}

From: ${ }^{1}$ Department of Physical Medicine and Rehabilitation, National Taiwan University Hospital, BeiHu Branch and National Taiwan University College of Medicine, Taipei, Taiwan; ${ }^{2}$ Department of Physical Medicine and Rehabilitation, China Medical University Hospital, Taichung, Taiwan; ${ }^{3}$ Department of Orthopedics, National Taiwan University Hospital and National Taiwan University College of Medicine, Taipei, Taiwan; ${ }^{4}$ Department of Anesthesiology, National Taiwan University Hospital and National Taiwan University College of Medicine, Taipei, Taiwan

Address Correspondence: Chih-Peng Lin, MD, PhD Department of Anesthesiology, National Taiwan University

Hospital and National Taiwan University College of Medicine, No.1, Changde St., Zhongzheng

Dist., Taipei City 10048, Taiwan (R.O.C.)

E-mail: cplino123@gmail.com

Funding Source: MOST-1052314-B-002-008. Conflict of interest: Each author certifies that he or she, or a member of his or her immediate family, has no commercial association (i.e., consultancies, stock ownership, equity interest, patent/licensing arrangements, etc.) that might pose a conflict of interest in connection with the submitted manuscript.

Manuscript received: 03-07-2016 Revised manuscript received: 03-31-2016 Accepted for publication: 04-04-2016

Free full manuscript: www.painphysicianjournal.com
Background: The suprascapular nerve accounts for $70 \%$ of shoulder sensory innervations, and suprascapular nerve block (SSNB) has been shown to be effective in the relief of chronic shoulder pain including rotator cuff tendinitis, subdeltoid impingement syndrome, and adhesive capsulitis. However, this remains inconclusive for patients undergoing surgery. The present meta-analysis aimed to explore the effectiveness of SSNB for relieving acute postoperative shoulder pain.

Objective: To explore the effectiveness of SSNB for relieving acute post-operative shoulder pain.

Study Design: A systematic review and meta-analysis.

Setting: Services of general surgery, orthopaedics, and anaesthesiology.

Methods: A systematic search of studies on SSNB for post-operative shoulder pain was conducted mainly in PubMed and Scopus. The standardized mean difference (SMD) of postoperative pain scales of SSNB versus placebo was treated as the primary outcome, whereas the odds ratio of nausea of SSNB versus placebo comprised the secondary outcome.

Results: The meta-analysis included 7 randomized controlled trials and 2 comparative studies comprising 681 participants in total. The quantitative analysis showed a significantly lower pain level of SSNB versus placebo in the shoulder surgery patient group (SMD: $-0.33 ; 95 \%$ confidence level $[\mathrm{Cl}]$ : -0.51 to -0.15$)$, but not in the non-shoulder surgery group (SMD: 0.28 ; $95 \% \mathrm{Cl}:-0.37$ to 1.93$)$. The pooled odds ratio of nausea in the SSNB arm compared with the placebo arm was 0.20 (95\% Cl: 0.09 to 0.45$)$, indicating a reduction in the incidence of nausea following SSNB.

Limitations: Heterogeneity of included trials.

Conclusions: SSNB significantly reduced acute post-operative shoulder pain in the shoulder surgery group but not in patients undergoing laparoscopic surgery or thoracotomy. This suggests that SSNB can be used as a method of polymodal analgesia for patients undergoing shoulder surgery; however, it is not recommended for the non-shoulder surgery patient population.

Key words: Suprascapular nerve, shoulder surgery, thoracotomy, laparoscopic surgery

Pain Physician 2016; 19:445-456 
T he suprascapular nerve accounts for motor innervations to the supraspinatus and infraspinatus muscles and $70 \%$ of shoulder sensory innervations, while the remaining $30 \%$ is managed by the axillary, supraclavicular, subscapular, medial pectoral, and lateral pectoral nerves $(1,2)$. Therefore, suprascapular nerve block (SSNB) is widely utilized in cases of recalcitrant shoulder pain, and its effectiveness at relieving chronic painful shoulders, including rotator cuff tendinitis, subdeltoid impingement syndrome, and adhesive capsulitis, has been demonstrated (3). The potential mechanism behind this pathology may be the amelioration of the neuropathic pain component or hydrodissection of an entrapped nerve $(4,5)$. However, although SSNB is an effective procedure for treating long-term shoulder pain, evidence relating to the relief of acute postoperative shoulder pain is lacking.

Acute post-operative shoulder pain can develop after shoulder surgery or after operations in nonshoulder regions. With the introduction of arthroscopic techniques, shoulder surgery has become less traumatic and painful. However, the magnitude of pain depends on surgical types. The most painful surgery is rotator cuff repair, whereas the shoulder instability restoration procedure is the least painful (6). Use of SSNB before and during shoulder surgery is theoretically effective for pain relief because the majority of pain generated is innervated by the suprascapular nerve. In contrast, surgeries such as laparoscopic surgery or thoracotomy can result in shoulder tip pain without direct influence on the shoulder joint. Irritation of the phrenic nerve due to pneumo-peritoneum and exploration of the pericardium or pleural surface is the most plausible mechanism $(7,8)$. The convergenceprojection theory implies a potential effect of SSNB at reducing post-operative shoulder pain through a shared pathway of the suprascapular and phrenic nerves above the cervical root level (9). However, the efficacy of SSNB in the non-shoulder surgery patient population remains inconclusive. Therefore, the present meta-analysis aimed to explore the effectiveness of SSNB in the relief of post-operative shoulder pain and to examine whether its effect differs between various surgical types.

\section{Methods}

\section{Search Strategy and Inclusion Criteria}

PubMed, Scopus, Cochrane Collaboration Central
Register of Controlled Clinical Trials, Cochrane Systematic Reviews, and ClinicalTrials.gov were searched for studies on the use of SSNB for post-operative shoulder pain relief, from the earliest record to January 2016 $(3,10,11)$. The bibliographies of included trials and related review articles were manually reviewed for relevant references. Literature not written in English or not available in full texts were excluded. We investigated studies employing SSNB for the relief of shoulder pain in patients receiving any type of surgery. The search strategy comprised the following keywords variably combined with SSNB: shoulder pain, post-operative pain, arthroscopy, surgery, and thoracotomy.

Regarding the types of included studies, we enrolled randomized controlled trials (RCTs) or comparative experimental trials, and excluded single-armed follow-up studies, case series, and case reports. All retrieved studies were required to comprise at least 2 treatment arms, one of which was SSNB and the other of which was placebo injection or no block. Since the present meta-analysis aimed to compare SSNB with placebo, the treatment arms using interscalene block or subacrominal infiltration or intra-articular injection of analgesics were not included in the quantitative analysis. The target population comprised patients who were at risk of developing post-operative shoulder tip pain, and the surgical region was not restricted to the shoulders. Post-operative shoulder pain was defined as shoulder pain within 72 hours after operations. The SSNB procedure could be conducted by using a single injection or continuous administration of local anaesthetics before or during the operation. Studies that explored the efficacy of SSNB for chronic shoulder pain or shoulder pain after stroke were beyond the scope of the present meta-analysis.

\section{Data Extraction and Quality Assessment}

Two reviewers examined all of the retrieved articles and extracted data using a predetermined form. We recorded the first author, year, sample size, number and type of treatment arms, participant characteristics, details of SSNB, comparative arm regimens, and summary of the general anaesthesia protocol. The methodological quality of enrolled studies was evaluated by 2 reviewers independently using Jadad scoring for the RCTs and the Newcastle-Ottawa Quality Assessment Scale for the comparative experimental trials. Jadad score evaluates the methodology of RCTs according to 3 aspects: randomization ( 2 points), blinding ( 2 points), and an account of all patients ( 1 point). The range of 
potential scores is 0 to 5 ; a higher score indicates better methodological quality (3). The Newcastle-Ottawa Quality Assessment Scale contains 9 items in 3 categories: participant selection (4 items), comparability (4 items), and exposure (3 items) (12). A study can be scored a maximum of one point for items in the Selection and Exposure domains and a maximum of 2 points for the Comparability domain. Between-reviewer discrepancies were solved through discussions under the supervision of the corresponding author.

\section{Data Synthesis and Analysis}

The standardized mean differences (SMDs) of post-operative shoulder pain between the SSNB and reference groups comprised the primary outcome (3). Data were extracted from the visual analogue scales evaluated at the rest position at the point closest to 24 hours post-surgery. A negative SMD value indicated SSNB to be a favorable treatment option. The odds ra- tios (ORs) of post-operative nausea in the SSNB group compared with the control comprised the secondary outcome (13). A random effects model was employed to pool individual SMDs and ORs; all analyses were performed using Stata 11.0 software (StataCorp, Texas, USA). Between-trial heterogeneity was determined by using 12 tests; values $>50 \%$ were regarded as considerable heterogeneity (13). Funnel plots and Egger's test were used to examine potential publication bias $(3,13)$. Statistical significance was defined as $P$-values $<0.05$, except for the determination of publication bias which employed $P<0.10$.

\section{Results}

\section{Study Search and Characteristics of Included Patients}

We retrieved 245 non-duplicate citations for a review of their titles and abstracts, and included 16

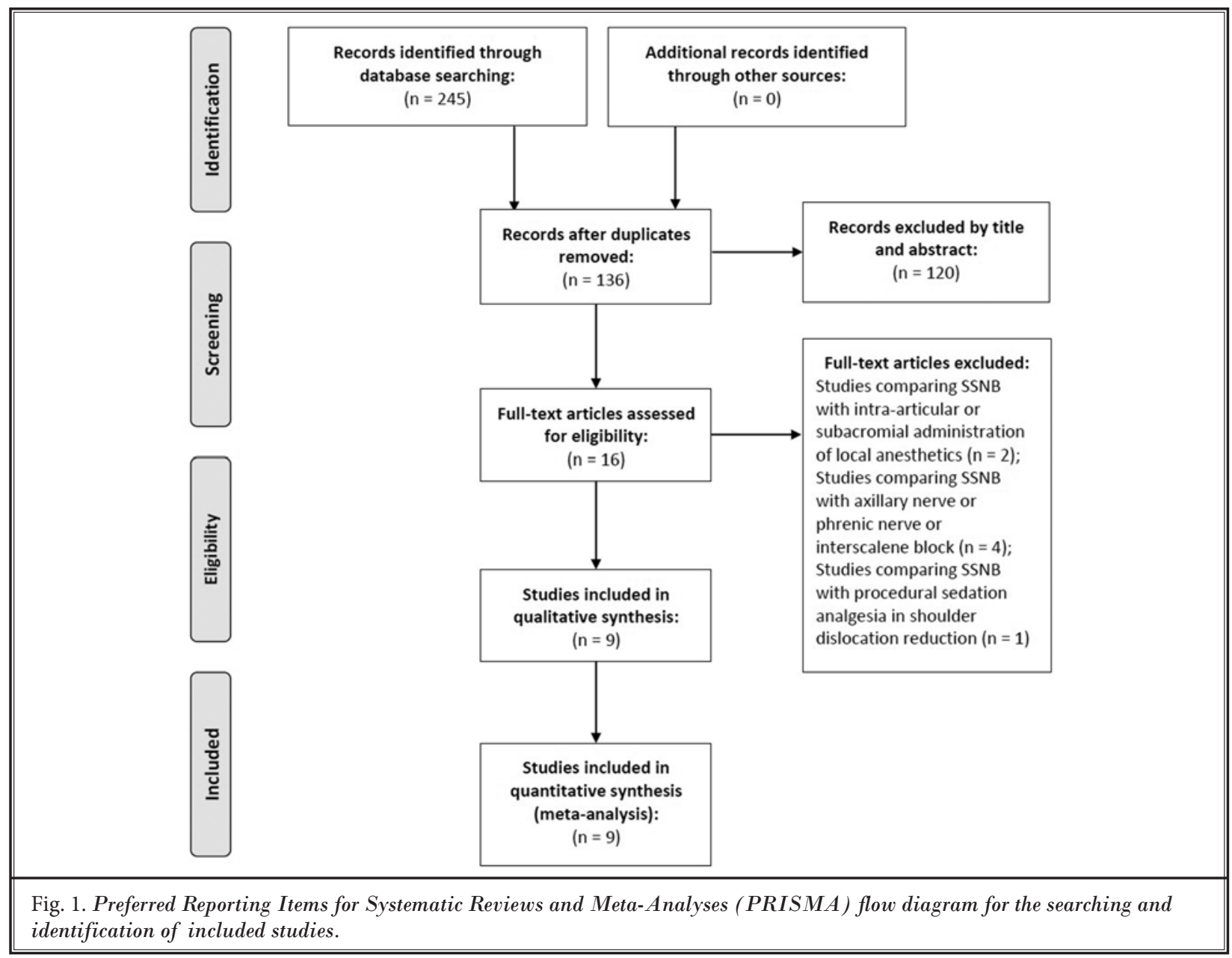


articles for meticulous evaluation after eliminating references violating the inclusion criteria (Fig. 1). We excluded 2 studies comparing SSNB with intra-articular or subacromial administration of local anaesthetics $(14,15), 4$ studies comparing SSNB with axillary nerve or phrenic nerve or interscalene block (16-19), and one study comparing SSNB with procedural sedation analgesia in shoulder dislocation reduction (20). Therefore, the meta-analysis included 4 two-armed RCTs (21-24), 2 three-armed RCTs $(25,26)$, one four-armed RCTs $(14)$, and 2 two-armed quasi-experimental studies $(27,28)$. In terms of the patient population, 6 trials targeted groups receiving shoulder surgeries $(14,21,23-25,28)$, 2 focused on participants undergoing thoracotomy $(22,27)$, and one investigated SSNB for shoulder tip pain after laproscopic surgery (26).

The final quantitative analysis included 681 participants. Two treatment arms in a four-armed RCT (14) and 2 treatment arms in 2 three-armed RCTs were not used for meta-analysis $(25,26)$. Patient age range was 24 to 72.6 years in the shoulder surgery group and 26.6 to 79 years in the non-shoulder surgery group. Diagnosis in the shoulder surgery group comprised subdeltoid impingement syndrome, rotator cuff tears, calcific tendinitis, and adhesive capsulitis. Regarding the nonshoulder surgery group, one trial recruited lung cancer patients for thoracotomy (27), while the remaining 2 did not specify the constitution of their patient population $(22,26)$. Patient characteristics, study methodology, and quality assessment of included trials are listed in Table 1, while Table 2 summarizes the SSNB procedures and general anaesthesia.

\section{SMDs of Post-operative Pain and Pooled Odds Ratio of Nausea}

The overall SMD of SSNB versus placebo regarding post-operative pain was -0.10 (95\% confidence interval [CI]: -0.53 to 0.32 ). The subgroup analysis showed a significantly lower pain level of SSNB versus placebo in the shoulder surgery group (SMD: $-0.33 ; 95 \% \mathrm{Cl}:-0.51$ to -0.15 ), but not in the non-shoulder surgery group (SMD: 0.28 ; $95 \% \mathrm{Cl}:-0.37$ to 1.93 ). Regarding SMD heterogeneity, the 12 was less than $0.01 \%$ in the shoulder surgery group and $93.5 \%$ in the non-shoulder surgery group (Fig. 2). The subgroup analysis based on different study designs and guiding techniques was listed in Table 3.

The pooled odds ratio of nausea in the SSNB arm compared with the placebo arm was 0.20 (95\% Cl: 0.09 -0.45 ), indicating a reduced incidence of nausea following SSNB. After removing a trial in the non-shoulder surgery group, the benefit of SSNB remained with a pooled odds ratio of 0.19 (95\% Cl: $0.08-0.45)$ (Fig. 3). Regarding the heterogeneity of odds ratio, the 12 was less than $0.01 \%$ in both the overall included studies and the shoulder surgery group. The Egger's test revealed the existence of significant publication bias $(P=0.089)$ regarding the overall SMD; however, the statistical significance reduced after being divided into both subgroups $(P=0.168$ in the shoulder surgery group and $P$ $=0.793$ in the non-shoulder surgery group). There was no publication bias detected in the overall odds ratio of nausea $(P=0.255)$. The funnel plots for SMD of postoperative pain and log odds ratio of post-operative nausea are shown in Figs. 4 and 5, respectively.

\section{Discussion}

The present meta-analysis focused on the use of SSNB for the relief of post-operative acute shoulder pain and nausea. It included 6 studies related to shoulder operations and 3 studies that recruited patients receiving thoracotomy or laparoscopic surgery. Compared with placebo, patients following SSNB presented with less shoulder pain in the shoulder surgery group, but the benefit was not significant in the non-shoulder surgery group. Similarly, there was a lower incidence of nausea after SSNB in the shoulder surgery group, although this beneficial effect was unclear in the nonshoulder surgery group due to the limited number of enrolled trials.

SSNB has been widely used in management of shoulder pain in miscellaneous conditions and there have been several systematic reviews and meta-analysis investigating this treatment. In a narrative review in 2011, Chan et al (29) investigated the anatomy of the suprascapular nerve, indications and techniques of SSNB, and outcomes of SSNB in the management of acute and chronic shoulder pain. This review found that SSNB may be beneficial for the control of post-operative pain after open and arthroscopic shoulder surgery, and reduces analgesic dosage and demand. However, conflicting results exist regarding the effectiveness of SSNB for shoulder pain management following thoracotomy. The most recent quantitative analysis of available trials regarding SSNB was published in 2015 (3). The article demonstrated the superiority of SSNB to placebo and physical therapy for relieving chronic shoulder pain; however, the patient population was out of the scope of our meta-analysis. Since post-operative shoulder pain drastically influences patient recovery and quality of life, it is of clinical importance to collect solid evi- 


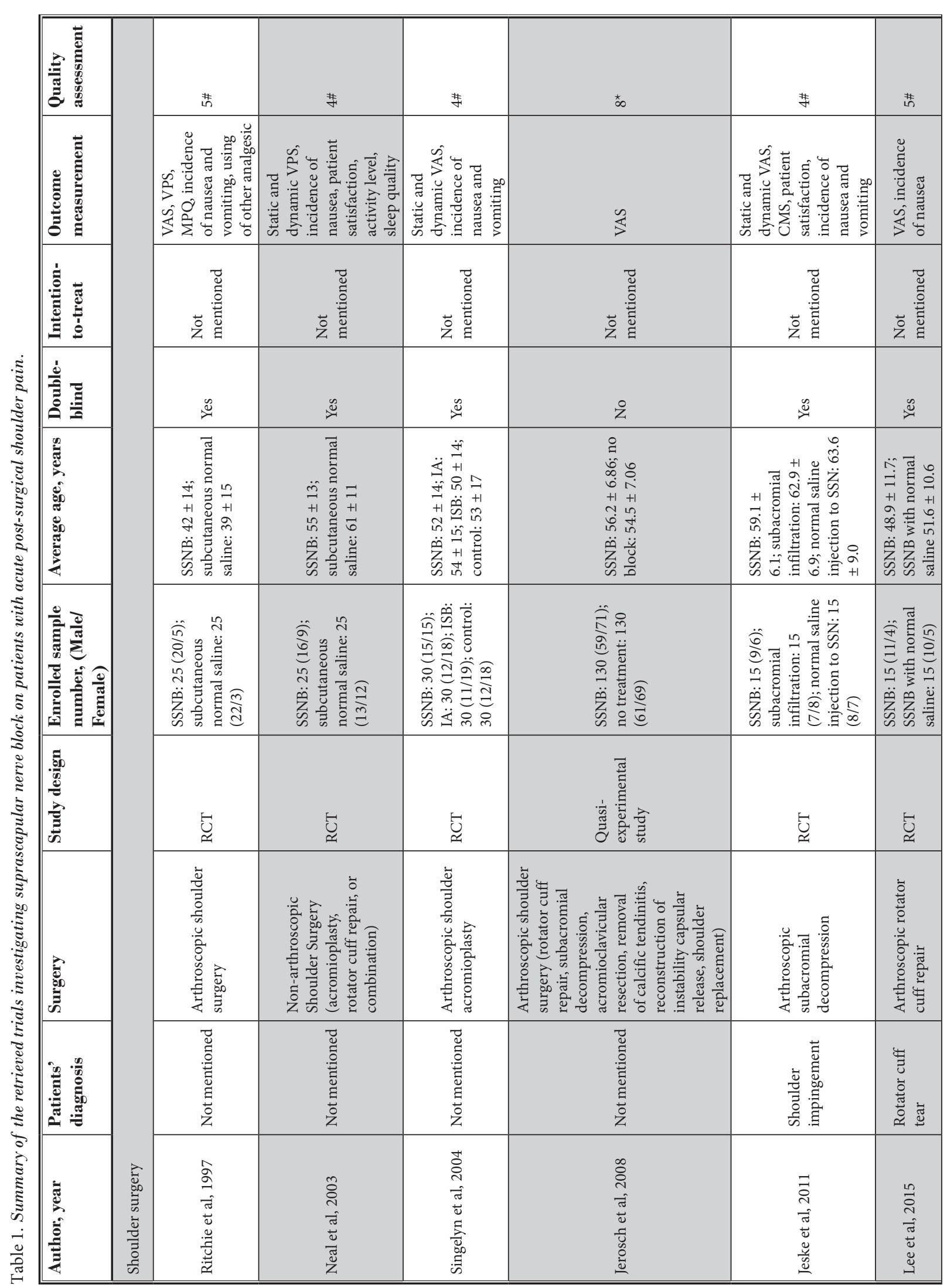



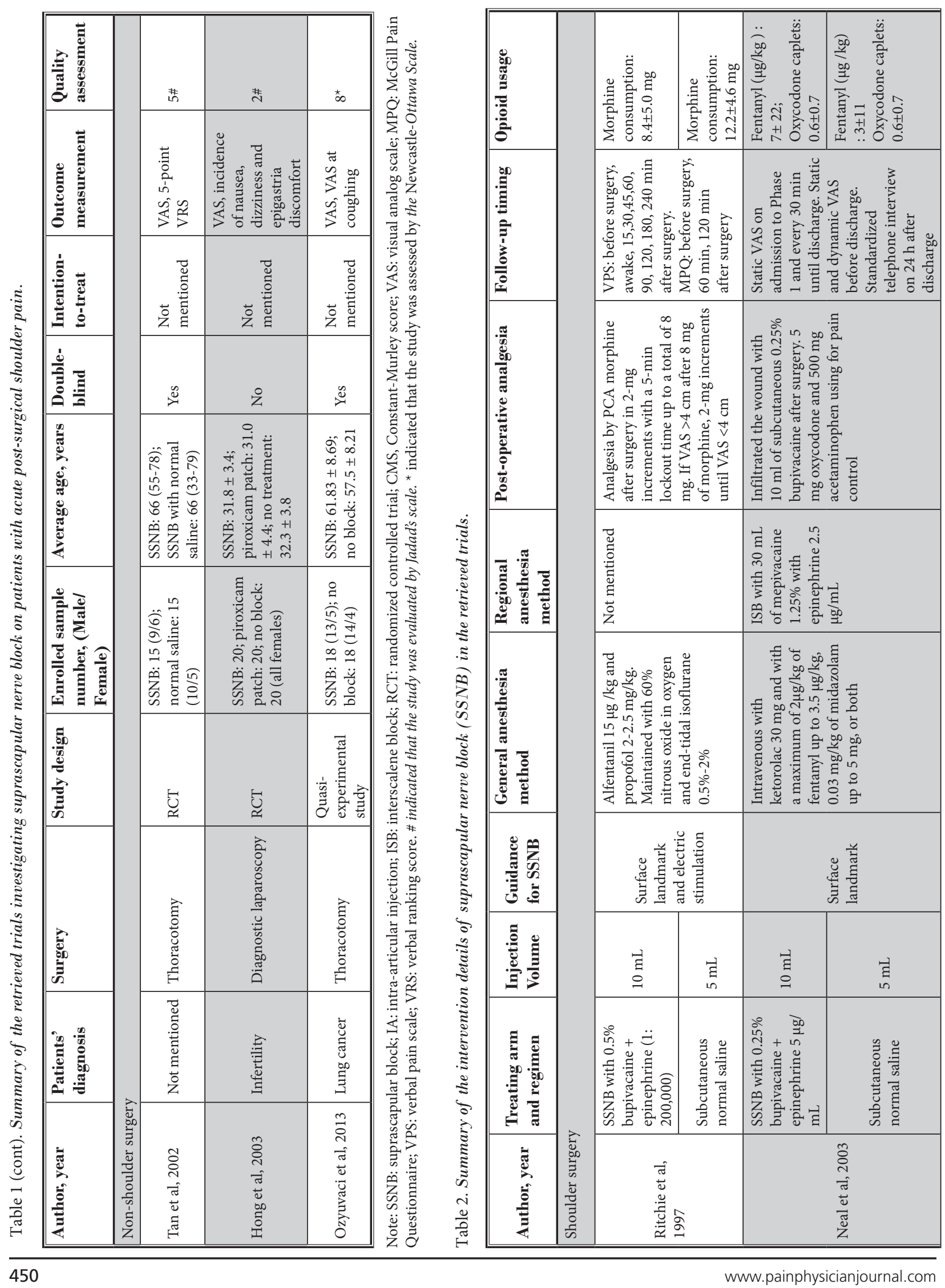


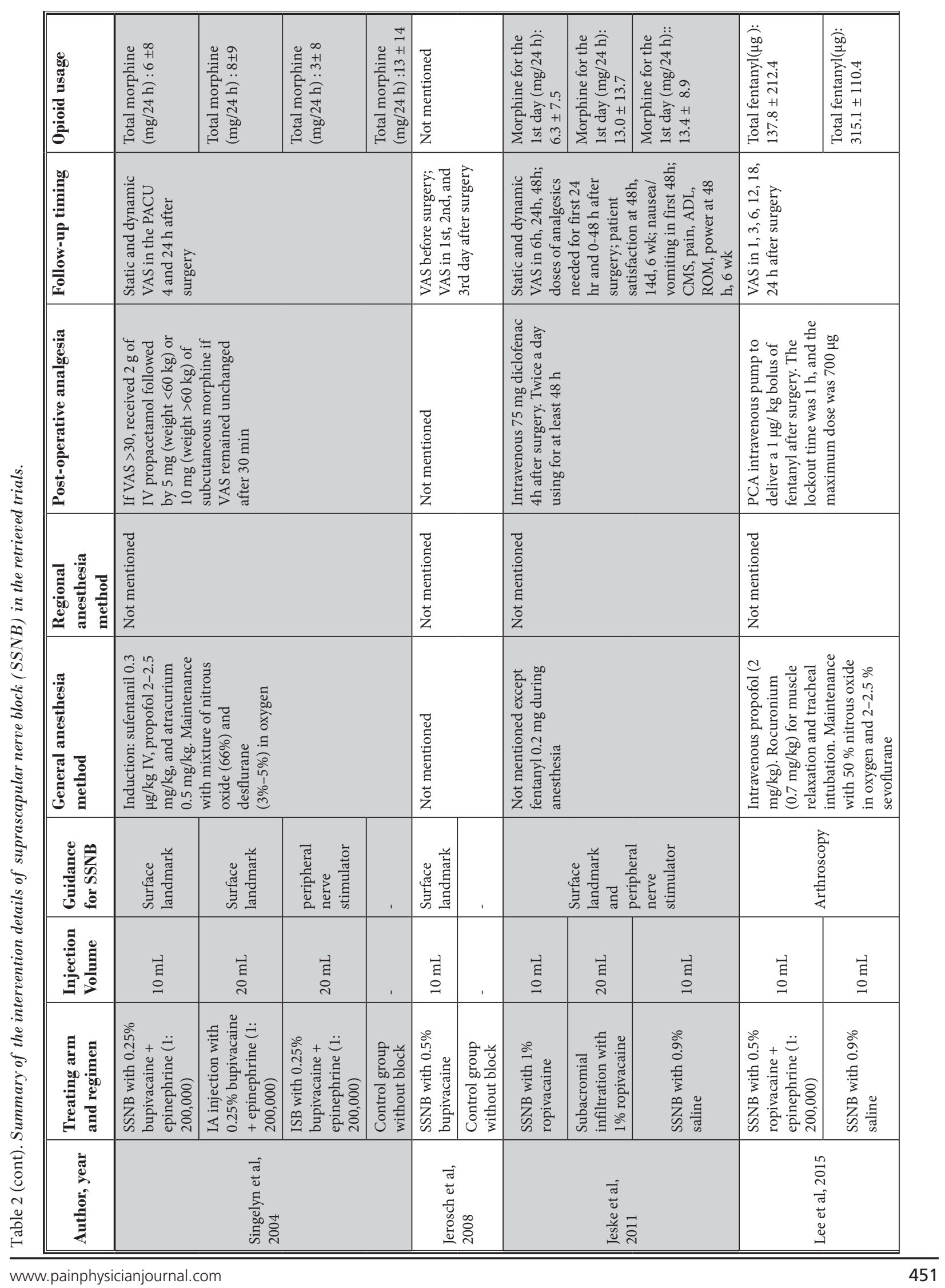




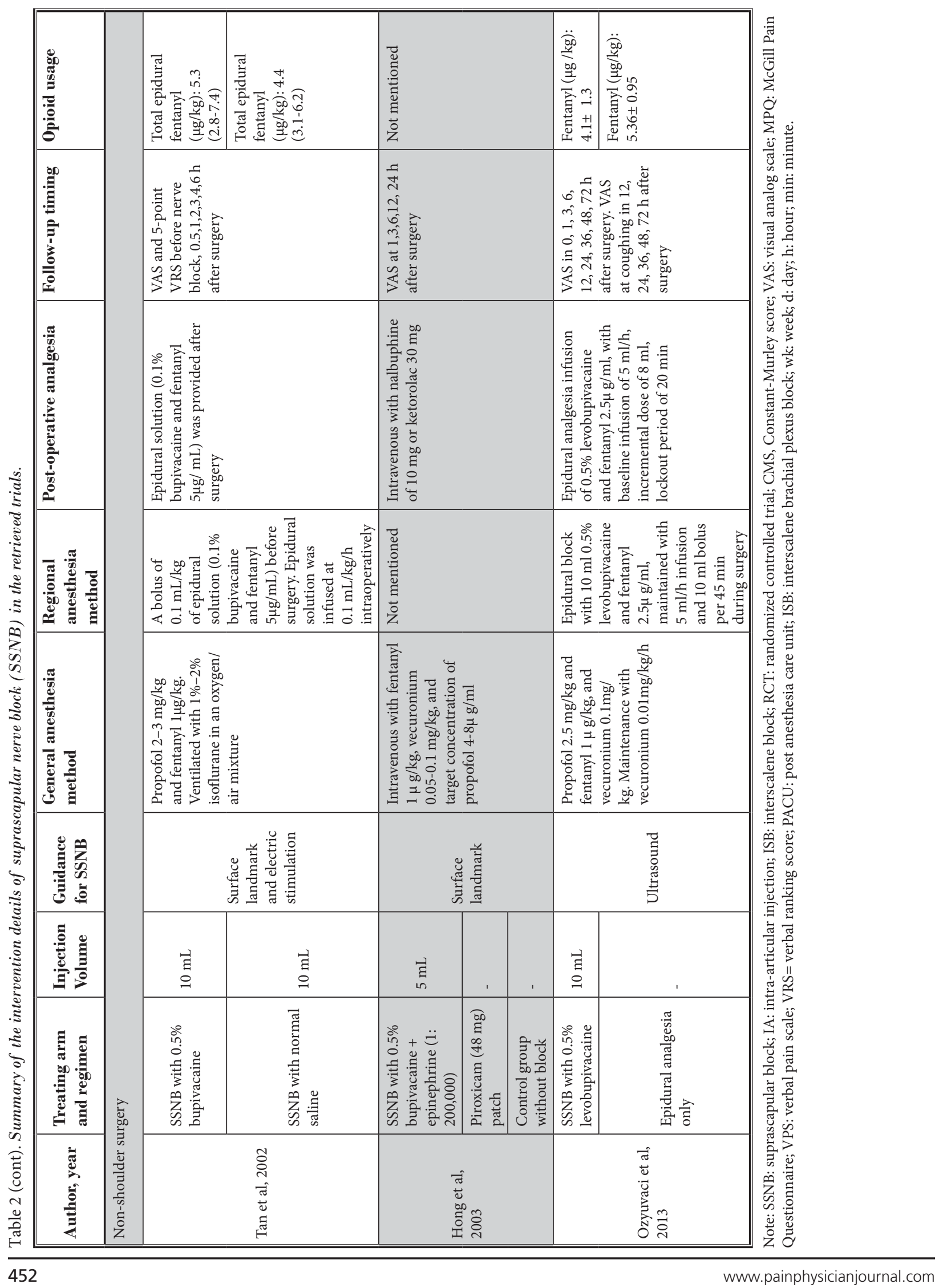


dence based on common statistical measures in terms of SSNB for shoulder pain relief after surgery.

The origin and mechanism of postoperative shoulder pain differs from that in chronic shoulder pain. In the shoulder surgery group, the magnitude of shoulder pain is related to the operative procedure (open versus arthroscopic surgery, type of surgery [rotator cuff versus non-rotator cuff], and causative factors of shoulder disorders [workrelated accidents, occupational overuse syndrome, or trauma or degenerative pathology]) $(6,30)$. The most painful period usually occurs on post-operative day 1 , when the effect of general anaesthesia gradually wears off and the tissues start to become swollen and edematous (6). Our results indicated that the use of SSNB resulted in a significantly less painful status in the shoulder surgery group compared with placebo. Since SSNB in most of the included studies was administered before or during surgery, its effect on post-operative day 1 was unlikely to be derived from the persistent action of local anaesthetics. We believe that the pre-operative and intraoperative implementation of SSNB could effectively reduce neurogenic inflammation, a neurally elicited local inflammatory response mediated by neuropeptides such as substance $P$ and calcitonin gene-related peptide (31). The benefit of SSNB was also reflected in the lower incidence of nausea, a common adverse symptom due to post-operative pain and use of opioid analgesics.

The cause of post-thoracotomy and laparoscopic surgery shoulder pain is presumed to differ from that after shoulder surgery. Irritation of phrenic nerves due to peritoneal stretching or exploration of the mediastinum and pericardium is a widely accepted mechanism (18). Since the suprascapular and phrenic nerves share the same origin (C5) in the root, SSNB may play a role in reducing shoulder-tip pain after operations. However, in the meta-analysis, there appeared to be inconsistent outcomes between trials; one of which showed a favorable result of SSNB, but 2 had an opposite effect, leading to a pooled SMD covering the zero value. One

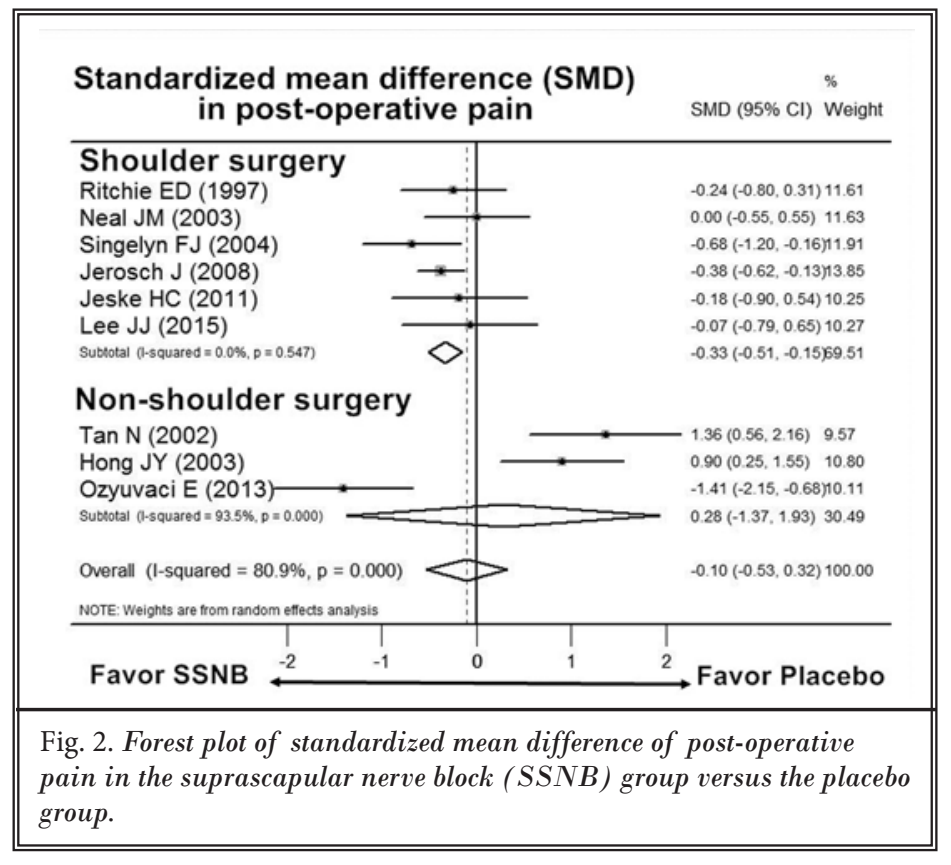

Table 3. Subgroup analysis of standardized mean differences based on study designs and guiding techniques for suprascapular nerve block

\begin{tabular}{|l|c|c|}
\hline \hline Subgroup & $\begin{array}{c}\text { Standardized } \\
\text { mean difference }\end{array}$ & $\begin{array}{c}\text { 95\% confidence } \\
\text { interval }\end{array}$ \\
\hline Study design \\
\hline Shoulder surgery group \\
\hline Randomized controlled trials & -0.27 & -0.54 to -0.00 \\
\hline Quasi-experimental studies & -0.38 & -0.62 to -0.13 \\
\hline Non-shoulder surgery group & 1.09 \\
\hline Randomized controlled trials & -1.41 & -2.15 to -0.68 \\
\hline Quasi-experimental studies & \multicolumn{2}{|l}{} \\
\hline Guiding technique & -0.53 to -0.16 \\
\hline Shoulder surgery group & -0.35 & -0.79 to 0.65 \\
\hline Surface landmark & -0.07 \\
\hline Arthroscopy & 1.09 \\
\hline Non-shoulder surgery group & -1.41 & -2.15 to -0.68 \\
\hline Surface landmark &
\end{tabular}

RCT indicated lower shoulder pain intensity after phrenic nerve infiltration than SSNB for post-thoracotomy pain (18). Therefore, direct targeting of the phrenic nerve may be a better solution for post-thoracotomy and -laparoscopy shoulder pain, and SSNB may not be a preferable pre-emptive analgesic procedure for non-shoulder surgeries.

Our subgroup analysis revealed that different study designs or guiding techniques did not result in discrepancy of treatment 


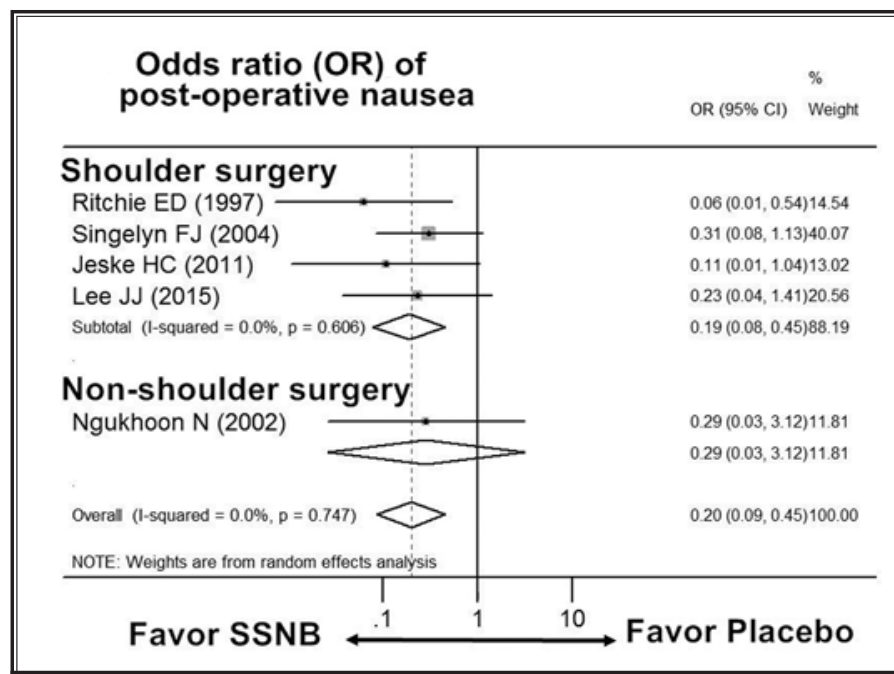

Fig. 3. Forest plot of odds ratio of post-operative nausea in the suprascapular nerve block ( $S S N B$ ) group versus the placebo group.

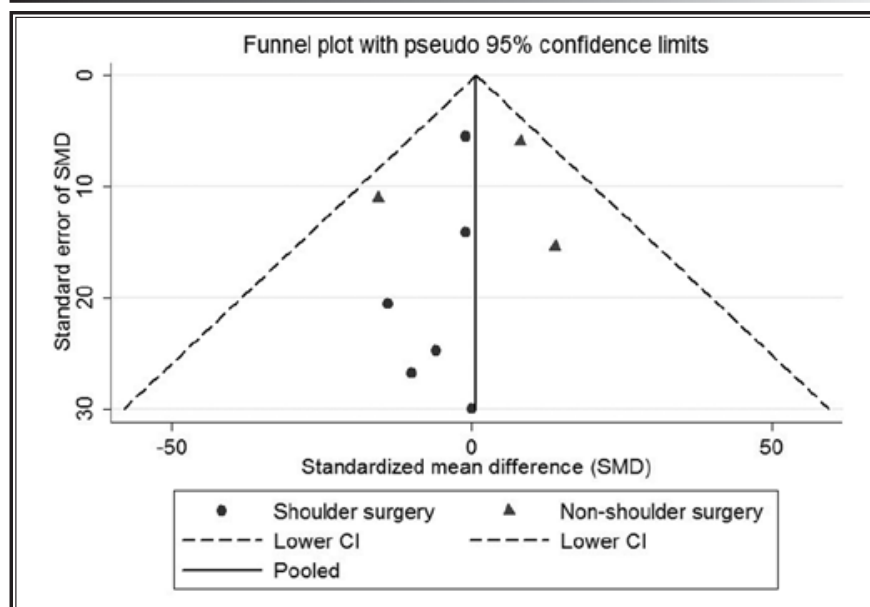

Fig. 4. Funnel plot of the standardized mean difference (SMD) of post-operative pain.

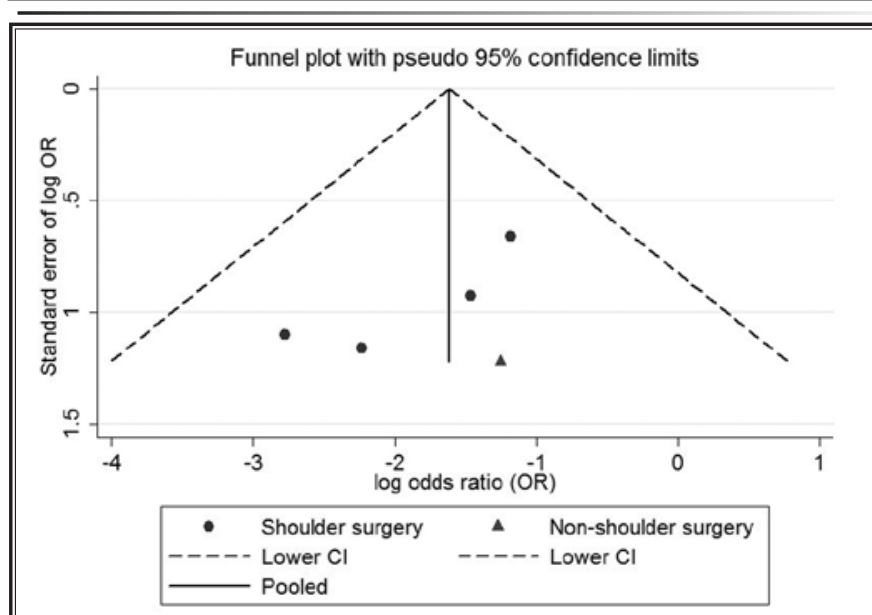

Fig. 5. Funnel plot of log odds ratio (OR) of post-operative nausea. effectiveness in the shoulder surgery group but led to a significant difference in the non-shoulder surgery group (Table 3 ). However, since only 3 studies were enrolled in the non-shoulder surgery group, the sample size was too small to claim that heterogeneity of effectiveness was derived from variations in study designs or guiding techniques.

There are several limitations of the present meta-analysis. First, the primary outcome was post-operative pain condition, not the change of visual analog scales pre- and post-operatively. The main reason for this was that only a minority of included trials recorded pre-operative pain status. Second, we did not serially investigate pain status; instead, we used the visual analog scale on the post-operative day 1 . This was because this time point was the most commonly documented in the results of the retrieved studies, especially in the shoulder surgery patient group. Another reason was based on a previous report stating that the patients felt the most pain 24 hours after arthroscopic shoulder surgery. Third, the outcome of post-operative pain can be modified by different analgesic regimens following surgery. Therefore, we also analyzed the odds ratio of the most prevalent adverse symptom, nausea, to examine whether there was inconsistency between both outcomes. Fourth, since SMD is derived from the betweengroup mean difference divided by the standard deviation, the value of SMD may be overestimated if the variability of the recruited population is artificially or accidentally reduced. In contrast, if the variability is increased, the SMD will be underestimated. Therefore, the researchers should consider the influence of measurement precision when reporting the treatment effectiveness by using the SMD. Finally, based on the above mentioned limitations, we suggest that future similar trials should document serial changes in post-operative shoulder pain and functional status as well as their pre-operative condition.

\section{Conclusions}

The present meta-analysis revealed that SSNB can lead to less painful shoulders for participants following shoulder surgery; however, its effectiveness is uncertain in patients receiving thoracotomy and laparoscopic surgery. SSNB 
also reduced the incidence of post-operative nausea. Therefore, our meta-analysis suggests that SSNB can be used as a method of polymodal analgesia for patients undergoing shoulder surgery, but is not recommended for patients undergoing non-shoulder surgery.

\section{Acknowledgment}

Funding was provided by the Ministry of Science and Technology (MOST-105-2314-B-002-008).

\section{References}

1. Vorster W, Lange CP, Briet RJ, Labuschagne BC, du Toit DF, Muller C), de Beer JF. The sensory branch distribution of the suprascapular nerve: An anatomic study.J Shoulder Elbow Surg 2008; 17:500-502.

2. Wu CH, Chang KV, Ozcakar L, Hsiao MY, Hung CY, Shyu SG, Wang TG, Chen WS. Sonographic tracking of the upper limb peripheral nerves: A pictorial essay and video demonstration. Am J Phy Med Rehabil 2015; 94:740-747.

3. Chang KV, Hung CY, Wu WT, Han DS, Yang RS, Lin CP. Comparison of the effectiveness of suprascapular nerve block with physical therapy, placebo, and intra-articular injection in management of chronic shoulder pain - a meta-analysis of randomized controlled trials. Arch Phys Med Rehabil 2016; In press.

4. Borstad J, Woeste C. The role of sensitization in musculoskeletal shoulder pain. Brazil J Phy Ther 2015; 19:251-256.

5. Chang KV, Hung CY, Ozcakar L. Snapping thumb and superficial radial nerve entrapment in De Quervain disease: Ultrasound imaging/guidance revisited. Pain Med 2015; 11:2214-2215.

6. Stiglitz Y, Gosselin O, Sedaghatian J, Sirveaux F, Mole D. Pain after shoulder arthroscopy: A prospective study on 231 cases. OTSR 2011; 97:260-266.

7. Imai $\mathrm{Y}$, Imai K, Kimura T, Horiguchi $\mathrm{T}$, Goyagi T, Saito H, Sato Y, Motoyama S, Nishikawa T, Minamiya Y. Evaluation of postoperative pregabalin for attenuation of postoperative shoulder pain after thoracotomy in patients with lung cancer, a preliminary result. Gen Thorac Cardiovasc Surg 2015; 63:99-104

8. Valadan M, Banifatemi S, Yousefshahi F. Preoperative gabapentin to prevent postoperative shoulder pain after laparoscopic ovarian cystectomy: A randomized clinical trial. Anesthesiol Pain Med 2015; 5:e31524.

9. Meyer-Witting M, Foster JM. Suprascapular nerve block in the management of cancer pain. Anaesthesia 1992; 47:626.

10. Hsiao MY, Hung CY, Chang KV, Chien
KL, Tu YK, Wang TG. Comparative effectiveness of autologous blood-derived products, shock-wave therapy and corticosteroids for treatment of plantar fasciitis: A network meta-analysis. Rheumatology 2015; 54:1735-1743.

11. Hsiao MY, Hung CY, Chang KV, Han DS, Wang TG. Is serum hypovitaminosis D associated with chronic widespread pain including fibromyalgia? A meta-analysis of observational studies. Pain Physician 2015; 18:E877-E887.

12. Chang KV, Hsiao MY, Chen WS, Wang TG, Chien KL. Effectiveness of intra-articular hyaluronic acid for ankle osteoarthritis treatment: A systematic review and meta-analysis. Arch Phy Med Rehabil 2013; 94:951-960.

13. Chang KV, Hung CY, Han DS, Chen WS, Wang TG, Chien KL. Early versus delayed passive range of motion exercise for arthroscopic rotator cuff repair: A metaanalysis of randomized controlled trials. Am J Sports Med 2015; 43:1265-1273.

14. Singelyn FJ, Lhotel L, Fabre B. Pain relief after arthroscopic shoulder surgery: A comparison of intraarticular analgesia, suprascapular nerve block, and interscalene brachial plexus block. Anesth Analg 2004; 99:589-592.

15. Yamakado K. Efficacy of arthroscopically placed pain catheter adjacent to the suprascapular nerve (continuous arthroscopically assisted suprascapular nerve block) following arthroscopic rotator-cuff repair. Open Access ] Sports Med 2014; 5:129-136.

16. Lee JJ, Kim DY, Hwang JT, Lee SS, Hwang SM, Kim GH, Jo YG. Effect of ultrasonographically guided axillary nerve block combined with suprascapular nerve block in arthroscopic rotator cuff repair: A randomized controlled trial. Arthroscopy 2014; 30:906-914.

17. Checcucci G, Allegra A, Bigazzi P, Gianesello L, Ceruso M, Gritti G. A new technique for regional anesthesia for arthroscopic shoulder surgery based on a suprascapular nerve block and an axillary nerve block: An evaluation of the first results. Arthroscopy 2008; 24:689-696.
18. Martinez-Barenys C, Busquets J, de Castro PE, Garcia-Guasch R, Perez J, Fernandez E, Mesa MA, Astudillo J. Randomized double-blind comparison of phrenic nerve infiltration and suprascapular nerve block for ipsilateral shoulder pain after thoracic surgery. Eur J Cardiothoracic 2011; 40:106-112.

19. Pitombo PF, Barros RM, Matos MA, Modolo NS. Selective suprascapular and axillary nerve block provides adequate analgesia and minimal motor block. Comparison with interscalene block. Brazil J Anesthesiol 2013; 63:45-51.

20. Tezel O, Kaldirim U, Bilgic S, Deniz S, Eyi YE, Ozyurek S, Durusu M, Tezel N. A comparison of suprascapular nerve block and procedural sedation analgesia in shoulder dislocation reduction. Am J Emerg Med 2014; 32:549-552.

21. Ritchie ED, Tong D, Chung F, Norris AM, Miniaci A, Vairavanathan SD. Suprascapular nerve block for postoperative pain relief in arthroscopic shoulder surgery: A new modality? Anesth Analg 1997; 84:1306-1312.

22. Tan N, Agnew NM, Scawn ND, Pennefather SH, Chester M, Russell GN. Suprascapular nerve block for ipsilateral shoulder pain after thoracotomy with thoracic epidural analgesia: A doubleblind comparison of $0.5 \%$ bupivacaine and $0.9 \%$ saline. Anesth Analg 2002; 94:199-202.

23. Neal JM, McDonald SB, Larkin KL, Polissar NL. Suprascapular nerve block prolongs analgesia after nonarthroscopic shoulder surgery but does not improve outcome. Anesth Analg 2003; 96:982-986.

24. Lee JJ, Yoo YS, Hwang JT, Kim DY, Jeon S), Hwang SM, Jang JS. Efficacy of direct arthroscopy-guided suprascapular nerve block after arthroscopic rotator cuff repair: A prospective randomized study. Knee Surg Sports Traumatol Arthroscopy 2015; 23:562-566.

25. Jeske HC, Kralinger F, Wambacher $M$, Perwanger F, Schoepf R, Oberladstaetter J, Krappinger D, Dallapozza C, Hoffmann F. A randomized study of the ef- 
fectiveness of suprascapular nerve block in patient satisfaction and outcome after arthroscopic subacromial decompression. Arthroscopy 2011; 27:1323-1328.

26. Hong JY, Lee IH. Suprascapular nerve block or a piroxicam patch for shoulder tip pain after day case laparoscopic surgery. Eur J Anaesthesiol 2003; 20:234-238.

27. Ozyuvaci E, Akyol O, Sitilci T, Dubus T, Topac Og lu H, Leblebici H, Ac Ikgoz A.
Preoperative ultrasound-guided suprascapular nerve block for postthoracotomy shoulder pain. Curr Ther Res Clin Exp 2013; 74:44-48.

28. Jerosch J, Saad M, Greig M, Filler T. Suprascapular nerve block as a method of preemptive pain control in shoulder surgery. Knee Surg Sports Traumatol Arthroscopy 2008; 16:602-607.

29. Chan CW, Peng PW. Suprascapular nerve block: A narrative review. Reg Anesth Pain Med 2011; 36:358-373.

30. Ruiz-Suarez M, Barber FA. Postoperative pain control after shoulder arthroscopy. Orthopedics 2008; 31:1130.

31. Lewis KM, Turner RJ, Vink R. Blocking neurogenic inflammation for the treatment of acute disorders of the central nervous system. Int J Inflam 2013; 2013:578480. 